\title{
Living on the edge: immune cells and immunopathology in the intestinal mucosa
}

\author{
Thomas Brunner
}

Published online: 3 June 2009

(C) Springer-Verlag 2009

The uptake and distribution of nutrients is a vital task of the gastrointestinal tract. Digested food is absorbed by intestinal epithelial cells and transported to underlying tissue from where it is distributed to the rest of the body. In order to optimize the nutrient transport from the intestinal lumen to the inside of our body, the epithelial surface is dramatically enlarged by macro- and mico-folds, villi, and finally the brush border on the absorptive epithelial cells, similar to a tree with branches and leaves. The simple single-cell layer architecture of the intestinal epithelium permits efficient uptake of nutrients and direct delivery to nearby blood vessels.

While this direct contact of our body to nutrients makes absorption more efficient, it also represents a risky business. The luminal side of the intestinal epithelial layer contains not only essential nutrients but also a plethora of commensal bacteria and potential pathogenic microorganisms. A disruption of this single-cell epithelial layer provides easy access of these microorganisms to the underlying tissue and eventually to the rest of the body, a process which may result in fatal bacteremia and has to be avoided by any costs. Not surprisingly, the intestinal mucosa is home to the largest and likely also most complex immune systems of our body with the difficult task to prevent invasion of the host by pathogens. Figure 1 illustrates the high density of different leukocyte subsets in the human colon, representing a complex and extensive immune cell defense line. Under most circumstances, the intestinal immune system seems to do quite a good job.

\footnotetext{
T. Brunner $(\bowtie)$

Division of Immunopathology, Institute of Pathology,

University of Bern,

Murtenstrasse 31,

3010 Bern, Switzerland

e-mail: tbrunner@pathology.unibe.ch
}

Although the single-cell epithelial layer is relatively leaky, in particular during inflammation, and specialized epithelial cells, so-called $\mathrm{M}$ cells, are even capable of transporting intact bacteria across the epithelial layer, invasion of the body by intestinal bacteria is relatively rare, demonstrating the effective protection by the local immune cells.

Efficient effector functions of local immune cells protect the host from invasion by pathogens. On the other hand, successful activation of immune effector cells often leads to inflammation-associated immunopathologies and bystander tissue damage. Various inflammatory diseases in humans demonstrate what happens when intestinal immune responses go over the top. It is currently well accepted that development of inflammatory bowel disease, i.e., ulcerative colitis and Crohn's disease, critically involves uncontrolled response of immune cells against commensal bacteria. Similarly, in food allergies and celiac disease, overreaction to otherwise harmless food antigens causes severe inflammation and associated pathologies. Chronic inflammatory responses in the gut may even result in the development of colonic cancer, e.g., as seen in many inflammatory bowel disease patients. It is currently not completely clear to what extent resident immune cells contribute to these pathologies and what is mediated by infiltrating inflammatory cells. Often, it may be even difficult to distinguish between them, as resident leukocytes under homeostatic conditions have a different phenotype than under inflammatory conditions, which may closely resemble that of their corresponding infiltrating counterparts. Detailed phenotypical and functional characterization of local intestinal immune cells is, thus, a must to understand their respective roles in health and disease. This phenotypic and functional adaptation is clearly evident when analyzing intestinal macrophages. As discussed in this issue, intestinal macrophages have maintained a high phagocytic activity, which is likely crucial for 
Fig. 1 Immune cells in the intestinal mucosa. Immunohistochemical detection (brown staining) of total leukocytes (CD45+), macrophages (CD68+), T lymphocytes $(\mathrm{CD} 3+)$, and $\mathrm{B}$ cells $(\mathrm{CD} 20+)$ in the same section of the human colonic mucosa

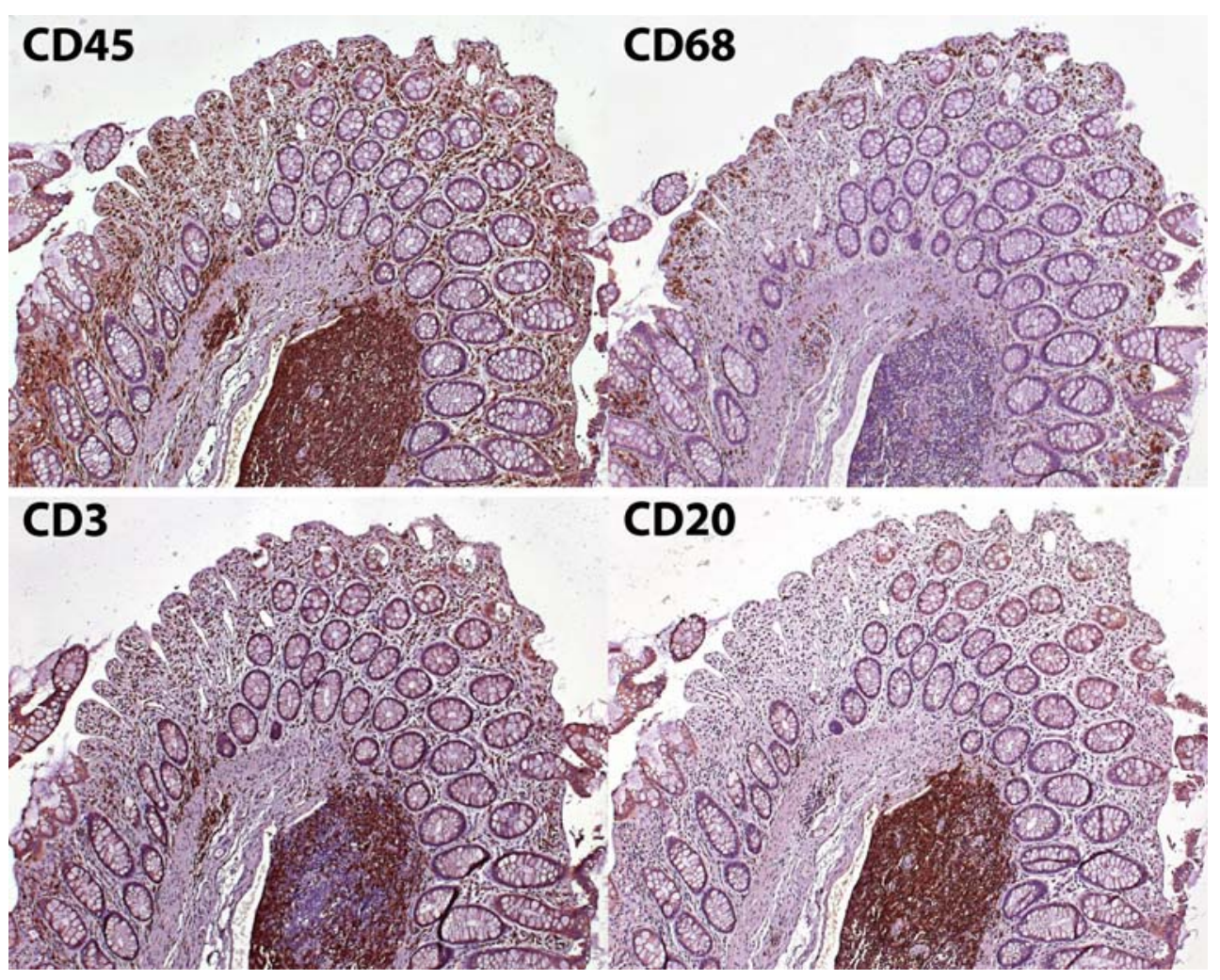

preventing bacterial spreading but lack many markers and cell surface receptors associated with the recognition of microorganisms, e.g., Toll-like receptors and immunoglobulin receptors. Clearly, this adaptation leads to a functional unresponsiveness to factors constantly present in the intestinal mucosa, thereby preventing uncontrolled and exaggerated immune responses.

Functional unresponsiveness of resident immune cells is, however, insufficient to prevent inflammatory responses against luminal antigens by infiltrating leukocytes. Physical and chemical damage of the intestinal epithelial layer will impair its barrier function. Thus, microbial products will further penetrate into the body and reach tissues where immune cells do not have such a functional adaptation as in the gut and may easily become activated by these potent triggers. When these primed and activated immune cells find their way to the source of antigen and microbial triggers, the associated inflammatory response will evidently lead to severe tissue damage, further access to luminal antigen, and initiation of a viscous cycle of inflammation. While this is likely the case in inflammatory bowel disease and maybe also other forms of intestinal inflammation, it is also clear that in the healthy individual, the activation of resident as well as infiltrating leukocytes is controlled at various levels, including regulatory $\mathrm{T}$ cells, suppressive cytokines, and other factors. Last but not least, presentation of antigen occurs in a "suppressed" environment, which educates immune cells to develop in a biased manner. Critically, under physiological conditions, intestinal immune cells of the adaptive immune system, i.e., $\mathrm{T}$ and $\mathrm{B}$ cells are not unresponsive to luminal antigen but rather develop immune effector function, which are generally not harmful to the tissue and its functions, e.g., synthesis of regulatory cytokines and switch to IgA-producing plasma cells.

This issue of Seminars in Immunopathology on the gastrointestinal immune system contains nine reviews discussing various aspects of mucosal immunology and immunopathology. Although given the size and complexity of the gastrointestinal immune system, this is more a glance than an in-depth overview; the different articles demonstrate the enormous developmental, functional, and regulatory adaptation of the intestinal immune cells to this peculiar environment. Finally, they make clear how difficult the appropriate balance between immune protection and immune tolerance in the gastrointestinal tissue can be, often deciding between war and peace. 